\title{
EL CONTENIDO EN LOS MEDIOS SOCIALES DE LOS DESTINOS TURÍSTICOS Y LA BÚSQUEDA DE INFORMACIÓN DE LOS USUARIOS ${ }^{1}$
}

\author{
Francisco Javier Paniagua* \\ Universidad de Málaga \\ Assumpció Huertas ** \\ Universidad Rovira i Virgili. Tarragona
}

\section{RESUMEN}

El objeto de este estudio es el análisis comparado de cómo los destinos turísticos utilizan los medios sociales y qué contenidos publican en su estrategia de comunicación; y cómo y qué buscan los usuarios cuando planifican un viaje. Las metodologías utilizadas son: entrevistas a directores de comunicación de destinos, encuestas a gestores de medios sociales, análisis de contenido de las publicaciones de los destinos y una encuesta a usuarios. Los resultados muestran ciertas diferencias en los medios sociales utilizados por ambos y en los contenidos publicados por los destinos y buscados por los usuarios, lo cual supone una interesante aportación para la comunicación de los destinos turísticos.

Palabras clave: marca de destino; comunicación; medios sociales; contenidos; usuarios.

Recibido: 30 de mayo de 2016

Devuelto para su revisión: 12 de diciembre de 2016

Aceptado: 1 de febrero de 2017

Departamento de Periodismo. Universidad de Málaga. C/ León Tolstoi, s/n. 29071 MÁLAGA (España). E-fjpaniagua@uma.es

** Departamento de Estudios de Comunicación, Universidad Rovira i Virgili. Avgda. Catalunya, 35. 43002 TARRAGONA (España).E-mail: sunsi.huertas@urv.cat

1 Este estudio es parte del proyecto CSO2012-34824 "Uso e influencia de los social media y la comunicación 2.0 en la toma de decisiones turísticas y en la imagen de marca de los destinos", que ha sido financiado por el Ministerio de Economía y Competitividad. Investigadora principal: Assumpció Huertas 
The content of tourist destinations in social media and the information search of users

\begin{abstract}
The aim of this study is the comparative analysis of how destinations use social media and what contents publish in their communication strategies and how do users use these social media and what do they look for in there when they organize a trip. The used methodologies are: interviews with communication directors of destinations, surveys to community managers, content analysis of the publications of the destinations and a survey to users. Results show some differences among social media used by both and among the contents published by destinations and looked for users. These results provide interesting insights for the communication of tourist destinations.
\end{abstract}

Keywords: destination brand; communication; social media; contents; users.

\title{
1. INTRODUCCIÓN
}

Los medios sociales han revolucionado la comunicación turística, y especialmente la comunicación de los destinos turísticos (Xian y Gretzel, 2010; Huertas, 2014). Debido a que en muchas ocasiones los destinos turísticos se desconocen por parte de los turistas potenciales con anterioridad a la decisión de visitarlos (Mill y Morrison, 2002), los comentarios y experiencias de terceros aportan mucha credibilidad a los usuarios (Xiang y Gretzel, 2010; Leung et al., 2013) y les influyen en sus decisiones turísticas (Schmallegger y Carson, 2010; Yoo y Gretzel, 2011).

Los medios sociales son importantes herramientas de relaciones públicas (Wigley y Lewis, 2012; Huertas, 2014), entendiendo las relaciones públicas como la gestión de la comunicación bidireccional entre una organización, en este caso un destino, y sus públicos (GrunigyHunt, 1984). Una de las principales potencialidades de los medios sociales es que permiten una efectiva comunicación de las identidades y las marcas de los destinos (Munar, 2011). Las marcas de los destinos son representaciones de su identidad que pretenden crear una imagen favorable en la mente de los públicos (Govers y Go 2009). A pesar de la relevancia de los medios sociales en la comunicación turística, todavía existen pocas investigaciones sobre el uso de los medios sociales por parte de los destinos turísticos (Huertas, Setó y Míguez, 2015) y sobre la influencia de los medios sociales en la comunicación de la marca de un destino turístico (Munar, 2011).

A través de los medios sociales los turistas pueden compartir sus experiencias de viaje y sus emociones (Henning-Thurau et al., 2004; Senecal y Nantal, 2004), lo cual ayuda a la creación de una imagen de los destinos y de su reputación (Inversini y Buhalis, 2009; Xiang y Gretzel, 2010; Marchiori y Cantoni, 2012). Además, la participación y comentarios de los turistas a través de los medios sociales comporta la adhesión emocional al destino, a su identidad y a su marca (Algesheimer, Dholakia, y Herrmann, 2005).

A través de su potencial interactivo los medios sociales permiten la creación de relaciones entre los usuarios y los destinos con sus marcas (Tussyadiah y Fesenmaier, 2009). Existen investigaciones que analizan la influencia de los medios sociales en la 
creación de la imagen de marca de los destinos y en las relaciones que crean los usuarios con estas marcas (Govers, Go, y Kumar, 2007; Laroche, Habibi y Richard, 2013; Stepchenkova y Zhan, 2013). Todos ellos coinciden en que a través de las conversaciones de los usuarios las identidades de los territorios se crean y se identifican con los usuarios (Govers y Go, 2003; Marine-Roig, 2013). Un estudio empírico basado en encuestas a los usuarios (Laroche et al., 2013) demostró que los medios sociales crean efectos positivos en las relaciones usuario-marca, que a la vez comporta una mayor credibilidad y lealtad de marca.

La marca de destino es un concepto que desde su surgimiento ha comportado un gran cambio en la comunicación de los destinos turísticos. Además, han proliferado muchos estudios sobre el tema desde diversas disciplinas científicas (Morgan, Pritchard y Piggott, 2003; Blain, Levy y Ritchie, 2005; Ekinci y Hosany, 2006; Choi et al, 2007), especialmente desde el turismo, el marketing y la comunicación. De hecho, los estudios sobre marca de destino son los más numerosos en investigación turística actual (Pike, 2002).

La principal aportación de las marcas de destino es la identificación de unos atributos con el territorio y la distinción entre destinos turísticos (Morgan, Pritchard y Piggott, 2003; Blain, Levy y Ritchie, 2005; Huertas, 2014). Por ello, los destinos tratan de comunicar una única identidad, una personalidad (Govers y Go, 2009) y unos valores que impacten en las emociones de los usuarios y hagan que se sientan atraídos por el lugar (Morgan, Pritchard y Piggott, 2003). Las marcas de destino, además, aportan un valor relacional con los públicos, ya que estos se identifican con la personalidad de la marca del lugar (Hankinson, 2004) y ello mejora la imagen del destino (Fournier, 1998), influye positivamente en las preferencias de los turistas potenciales (Sirgy, 1982) y aumenta las decisiones turísticas favorables en la elección de destino (Gallarza et al., 2002; Pike y Ryan, 2004; Ekinci y Hosany, 2006; Choi et al, 2007). Consecuentemente, es necesario conocer cómo los destinos turísticos comunican sus marcas, especialmente a través de los medios sociales, con el objetivo de que esta comunicación sea eficiente y útil para los turistas.

\section{ESTADO DEL ARTE}

Existen diversos estudios realizados sobre comunicación de las marcas de destino y sobre el rol de los medios sociales en dicha comunicación. Un primer tipo de estudios se centra más en la gestión de la comunicación de las OMDs (Organización de Marketing de los Destinos) y el rol de los medios sociales (Roque \& Raposo, 2015) y un segundo tipo, en la comunicación de las marcas a través de dichos medios (Kasemsap, 2016). Y dentro de este segundo tipo de estudios, los hay basados en demostrar la importancia de los medios sociales en la comunicación de las marcas de destino y otros centrados en el análisis de la comunicación de marca que las OMDs realizan.

Dentro del primer tipo de estudios, Roque y Raposo (2015) han analizado una serie de destinos turísticos nacionales y han comparado el uso de los medios sociales observando la existencia de similitudes en su uso, aunque también han detectado diferencias según el tipo de destino. En general, constatan que los medios sociales más utilizados son Facebook, Twitter y Youtube, siendo Facebook el que genera más seguidores y mayor interacción con los usuarios. 
Los estudios basados en el rol de los medios sociales, como este de Roque y Raposo (2015), muestran que mientras estos medios van adquiriendo popularidad, los usuarios también van ganando poder (Thevenot, 2007), y en cambio las DMOs van perdiendo influencia hasta el punto que ya no poseen el control de la imagen del destino (Hays, Page y Buhalis, 2013).

Por otro lado, dentro del segundo tipo de estudios, basados en la comunicación de las marcas de destino a través de los medios sociales, algunos demuestran que estos medios tienen un especial potencial para la comunicación de las marcas (Kasemsap, 2016). Habibi et al. (2014) afirman que los gestores de marcas deben entender cómo operan las marcas en los medios sociales para sacar el máximo rendimiento en su comunicación. Una de las estrategias más utilizadas en los medios sociales es la creación de comunidades de marca, que ayudan a la creación de relaciones entre la marca y los consumidores. Además, la co-creación de contenidos en los medios sociales genera relaciones de los usuarios con las marcas y todo ello aumenta la lealtad hacia la marca (Algesheimer et al., 2005) y las conexiones con ella (Zaglia, 2013). Así pues, cuando se crean comunidades de marca con relaciones con los consumidores estos pueden compartir sus experiencias en los medios sociales y ello también aumenta la lealtad hacia las marcas (Brodie et al., 2013).

Finalmente, hay un segundo tipo de estudios dentro de los de comunicación de marcas de destino que se centra en el análisis de la comunicación realizada a través de los medios sociales, como por ejemplo el estudio de Dickinger y Lalicic (2016), que analiza la personalidad de marca de los destinos reflejada en los comentarios de los usuarios en Tripadvisor.

El estudio de Dickinger y Lalicic (2016), al igual que el nuestro, destaca la importancia de la comunicación de la marca con sus dos componentes principales (atributos y valores emocionales), que deben ser claves en la comunicación de los destinos turísticos a través de los medios sociales. La mayoría de trabajos académicos sobre las marcas de destino, desde los inicios hasta la actualidad (Baloglu y McCleary, 1999; Echtner y Ritchie, 2003; Hosany, Ekinci y Uysal, 2006; Huertas, 2014), afirman que estas se componen de dos principales elementos: unos atributos tangibles, que equivaldrían a los elementos cognitivos, y unos valores emocionales o elementos afectivos.

Cabe destacar que, generalmente, los estudios demuestran una dominancia de la comunicación de los atributos tangibles en detrimento de la comunicación de los valores emocionales (Bigné, García y Blas, 2009; Michaelidou et al., 2013). Una investigación reciente de Míguez y Huertas (2015) sobre el uso de las imágenes en la comunicación de la marca de destino a través de los medios sociales demostró que las fotografías poseían un gran potencial en la identificación y comunicación de los valores emocionales, pero curiosamente, los destinos utilizaban las fotografías en mayor medida para comunicar atractivos turísticos del territorio que valores emocionales de marca.

Diversos estudios han demostrado que la interactividad de los espacios web influye positivamente en la comunicación de la identidad, la marca de los territorios, ya que la interactividad comporta mayor tiempo de navegación e involucramiento de los usuarios y mejor imagen de marca y lealtad hacia ella. Pero muchos estudios también han demostrado que los destinos infrautilizan el potencial interactivo de los medios sociales en la comunicación de la marca territorio (Huertas, Setó y Míguez, 2015; Huertas y Mariné-Roig, 2015). 
Debido a las potencialidades de los medios sociales para la comunicación de los destinos y sus marcas, los gestores de la comunicación de los destinos los utilizan cada vez más, pero en ocasiones con gran desconocimiento sobre su uso, sin seguir una planificación estratégica (Hvass y Munar, 2012) y sin sacar provecho de todas sus potencialidades (Huertas, Setó y Míguez, 2015). El uso de los medios sociales entre los destinos es, en gran medida, experimental, y sus estrategias varían de forma significativa (Hays, Page y Buhalis, 2013).

Así pues, los objetivos de este estudio son: 1-conocer si los destinos turísticos españoles utilizan todas las potencialidades de los medios sociales como canales de relaciones públicas para crear diálogo con sus públicos y generarse una buena imagen, 2-analizar si comunican a través de los medios sociales realmente su identidad y su marca, con sus dos componentes principales por igual, y finalmente 3-conocer qué informaciones buscan los usuarios en los medios sociales y compararlo con la comunicación de los destinos en estos medios para ver si son coincidentes y si existe una eficaz estrategia de comunicación 2.0.

\section{METODOLOGÍA}

Para cumplir estos objetivos de investigación el estudio se estructuró en 3 fases y se utilizaron 4 metodologías de análisis.

\section{Figura 1 \\ FASES DEL ESTUDIO Y METODOLOGÍAS DE ANÁLISIS UTILIZADAS}

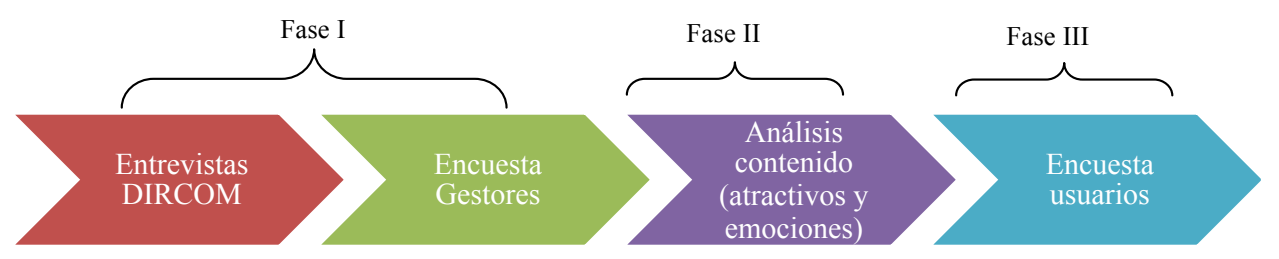

Fuente: elaboración propia.

\subsection{Fase I: entrevistas a los Directores de comunicación y encuestas a los Gestores de los medios sociales}

En la primera fase, para conocer cómo planifican su estrategia de marca los destinos, qué contenidos comparten en los medios sociales y si utilizan todas las potencialidades interactivas de estos medios para crear diálogo y buena imagen con los públicos, se utilizaron dos técnicas metodológicas: entrevistas en profundidad a los directores o responsables de comunicación de los destinos y encuestas online a los gestores de los medios sociales.

Primero, se realizaron las entrevistas en profundidad a 8 directores de comunicación de diferentes destinos turísticos españoles de la muestra del estudio que fuesen representativos de las diversas áreas geográficas y uno de cada tipo de destino.

La muestra de destinos analizada en el estudio se estructuró en 38 destinos turísticos españoles de 5 comunidades autónomas, las más representativas de todo el territorio 
español, que corresponden a las Áreas Nielssen en investigación de mercado: Andalucía, Islas Canarias, Cataluña, Galicia y Madrid. Por cada Comunidad se seleccionaron 7 tipos distintos de destino: comunidad autónoma, gran municipio o capital de la comunidad autónoma, gran destino de costa, ciudad patrimonio de la humanidad, destino litoral, destino de interior, ciudad media y destino de alta montaña. Esta tipología de destinos se ha recogido del Manual de Modelos de Gestión de Turismo Local del Ministerio Español de Industria, Turismo y Comercio (FEMP y TourSpain, 2008), por ser representativa de todos los tipos de destino españoles. Así pues, se seleccionó un tipo de destino por cada comunidad cuando las características geográficas lo permitieron.

El objetivo de las entrevistas en profundidad a los directores de comunicación era conocer qué estrategias comunicativas y de contenido poseían y qué directrices daban a los gestores de los medios sociales para la comunicación de dichos destinos. Por ello, el guión se estructuró en 4 bloques de preguntas: 1-funciones y estructura organizacional, 2-presencia del destino en los medios sociales, 3-uso interactivo y relacional y 4-credibilidad e impacto de los medios sociales.

La segunda metodología utilizada fue la encuesta online a los gestores de los medios sociales de los destinos de la muestra. Se envió a todos los destinos de la muestra y se obtuvo un total de 16 cuestionarios válidos, representando un $44 \%$ de la población objeto de estudio. La recogida de los datos a través de la encuesta se realizó durante la primera quincena del mes de octubre de 2014. Los resultados se trataron de forma agregada y sin distinción por tipología de destinos. El diseño del cuestionario se basó en cuatro bloques: 1-contexto del puesto de trabajo, 2-las funciones realizadas por el responsable de la comunicación digital, 3-la formación recibida y 4-gestión, presencia y estrategia comunicativa en los medios sociales. En este apartado se analizaron las cuestiones relacionadas con la difusión de la marca destino.

\subsection{Fase II: Análisis de contenido de los medios sociales}

En la segunda fase del estudio, para conocer la comunicación de la marca realizada por los destinos españoles, con sus atractivos y sus valores emocionales, realizamos un análisis de contenido de las últimas 25 publicaciones de cada uno de sus medios sociales oficiales. Se recogieron un total de 2.759 publicaciones: 943 en Facebook, 781 en Twitter, 542 en Youtube, 329 en Flickr y 155 en blogs.

Para llevar a cabo el análisis de contenido de los medios sociales nos centramos en los dos elementos que configuran las marcas de destino: uno funcional o tangible y otro emocional (Baloglu y McCleary, 1999). Los elementos funcionales son los atractivos turísticos que tienen los destinos y los elementos emocionales son los valores que representan su personalidad. Para analizar los elementos funcionales tomamos algunos listados utilizados por diversos autores anteriormente (Beerli y Martin, 2004; Wenger, 2008; Marina-Roig, 2013). Estos elementos son: naturaleza, ocio, cultura, patrimonio tangible, patrimonio intangible, transporte, alojamiento, actividades/escapadas, compras, sol y playa, deporte, tecnología, servicios, etc. No obstante, para analizar la comunicación de marca de destino también tuvimos que analizar si se comunicaban los valores emocionales y utilizamos 
la Brand Personality Scale de Aaker (1997), que ya ha sido también utilizada por otros autores para el análisis de las marcas de destino (De Moya y Jain, 2013; Huertas, 2014).

En general, los estudios previos se han centrado en investigar el componente funcional de las marcas y han dejado de lado los valores emocionales (Ekinci y Hosany, 2006). Por este motivo, el presente estudio también incluye el estudio de los valores de las marcas de los destinos estudiados.

El equipo de investigadores realizó el análisisdel contenido de los mensajes manualmente después de leerlos y comprobó que el contenido estaba siendo clasificado y codificado de la misma manera.

\subsection{Fase III: Encuesta a usuarios}

Finalmente, en la tercera fase de la investigación se realizó una encuesta online a través de un panel NETQUEST a usuarios, el público objetivo de la cual eran residentes en España que hubiesen realizado un desplazamiento con fines turísticos en los últimos dos años y para ello hubiesen consultado Internet. La muestra seleccionada fue de 800 usuarios estratificados por cuotas de género, edad y área geográfica (areas Nielsen). Esta se realizó del 24 de abril al 4 de mayo de 2015.

Los resultados, que se muestran a continuación, se han organizado en tres grandes bloques o epígrafes según las fases de la investigación. El primero analiza cómo planifican los diferentes destinos turísticos su estrategia de marca en su comunicación a través de los medios sociales. El segundo muestra qué comunican: atractivos turísticos y valores emocionales. Y el tercero analiza qué informaciones buscan realmente los usuarios en los medios sociales cuando planifican un viaje con el objetivo de comparar lo comunicado por el destino con lo buscado por el viajero.

\section{RESULTADOS}

\subsection{Gestión de la marca destino en los medios sociales (a partir de las entrevistas a los directores de comunicación y las encuestas a los gestores de los medios sociales)}

Respecto a la gestión de la marca y las directrices para la creación de los contenidos en los medios sociales, las entrevistas a los directores de comunicación de los destinos mostraron que en todos los casos tienen establecidos diferentes métodos de coordinación. Los ocho destinos estudiados cuentan con diferentes protocolos de actuación: un Plan Estratégico de Turismo y diversos Planes de Comunicación y Marketing. Estos documentos y la lógica definen la estrategia comunicativa, mientras que las formas se recogen en los manuales de estilo o de uso de la comunicación en los medios sociales. La OMD de una capital de comunidad autónoma, por ejemplo, tiene definidos el contenido y la estrategia en el Plan Estratégico de la Ciudad primero y a partir de ahí en el Plan de Comunicación y el Manual de uso de la Comunicación en las Redes Sociales. Otra de ellas cuenta también con un Plan Estratégico de Turismo en el que se especifican los contenidos. En otra, la oficina de Comunicación de la Empresa Pública de Turismo se encarga fundamentalmente de la investigación de tendencias y la observación del entorno. En este caso, la forma más 
utilizada para definir la hoja de ruta tanto estratégicamente como en contenidos son las reuniones. Finalmente, en otra es el Plan de Marketing el documento base que define la planificación de la marca de destino a medio-largo plazo, con la finalidad de que no le afecten los cambios políticos.

Facebook, Twitter, Youtube, Pinterest, Instagram, Foursquare y Google + son los principales medios sociales en los que tienen presencia los destinos estudiados, y por este orden. Las razones por las que se eligieron estos canales son fundamentalmente para mejorar la difusión de la marca, para tener seguidores o fans y porque es una comunicación que requiere una menor inversión que las tradicionales. Tan solo uno de los entrevistados destacó como motivo de uso las posibilidades que ofrecen los medios sociales como herramientas de investigación y observación del entorno, de la competencia o de los usuarios finales y sus opiniones. Igualmente, alguno de los entrevistados resalta el cambio del modelo de comunicación, en el que actualmente el objetivo final no debe ser ofrecer información, si no la comunicación y la creación de diálogo con los usuarios. Finalmente, coinciden que en la web 2.0 los conceptos de publicar y publicitar se ven sustituidos por participar y socializar la información.

En general, todos los entrevistados son conscientes de los beneficios que aportan los medios sociales al destino, entre los que destacan la ampliación de la visibilidad del lugar, sus acontecimientos, su agenda, el seguimiento de las opiniones de los usuarios y las posibilidades de segmentación a la hora de difundir información y de analizar los resultados. Solo dos destinos de los ocho preguntados van más allá, y resaltan también las posibilidades de interaccionar con los usuarios y la posibilidad de escucharlos a través de estos canales de comunicación. La mayoría de los responsables de comunicación entrevistados consideran que es importante contar con una buena planificación y profesionalización para gestionar la presencia de una marca de destino en los medios sociales.

Como norma general, los destinos estudiados se proponen para su estrategia digital objetivos cuantitativos (como incrementar el número de seguidores y ganar visibilidad) y en algunos casos la interacción y el valor cualitativo de las opiniones sobre la marca, así como la segmentación de los mensajes y los análisis. Hay algún destino que apuesta más por crear opinión en los usuarios e interaccionar con estos, porque así se convierten en cómplices en la difusión de los contenidos a través de los medios sociales. Uno de los Directores de comunicación entrevistados destacó que:

"El objetivo no es contar con el mayor número posible de fans o seguidores, sino tener una masa crítica que interactúe con nosotros y difunda nuestros contenidos, tanto a través de redes sociales, como sobre todo en sus conversaciones con amigos, familiares, conocidos o incluso clientes. Es decir, nuestro objetivo es convertirnos en fuente de información primaria, en información con posible interés turístico tanto para ciudadanos y los posibles visitantes, como para profesionales del sector (por ejemplo, agentes de viajes, touroperadores, periodistas especializados, etc." (Entrevistado 3)

Mientras que otros centran sus objetivos en la reputación digital del destino en todos sus niveles y lograr influencia: 
“...Ser como más influyentes y buscar la llave de cierto reconocimiento profesional, es decir, en el
ámbito corporativo se trata de subir la reputación general del consorcio, de la empresa y ayudar a
la comercialización de nuestros productos, y a nivel promocional llegar a cuanta más gente mejor
de todo el mundo y cada vez hacerlo mejor, con más contenidos específicos en idiomas, en estos
mercados interesantes y el siguiente paso debe ser crecer con más idiomas”. (Entrevistado 4)

Para lograr mayor visibilidad de las plataformas o perfiles oficiales de los medios sociales, todos los destinos incluyen información y enlaces de los mismos en cualquier herramienta de comunicación como folletos, comunicados de prensa o en las páginas web en las que hay enlaces y widgets.

En lo que respecta a los públicos, tres de los destinos analizados apuestan por enfocar sus mensajes en los medios sociales tanto a sus públicos internos (residentes, empresarios locales, medios de comunicación) como a los externos (oficinas de turismo, medios de comunicación, turistas o profesionales del sector). El resto, en cambio, opta por focalizar sus esfuerzos en el usuario final, es decir, en el turista potencial. La agenda cultural, el patrimonio, la historia, la gastronomía, reportajes de interés, newsletters, informaciones, fotografías y vídeos son los principales contenidos que comparten en los medios sociales los destinos y los propios turistas, que a la vez contribuyen al contar sus experiencias en la difusión de la marca territorio.

En lo que respecta al grado de apertura y la permisividad a los públicos, con los comentarios negativos, por ejemplo, impera el sentido común y en general no hay unas directrices establecidas al respecto. No obstante, en todos los casos analizados los comentarios negativos se eliminan si no son respetuosos o si se trata de información comercial. Todos los entrevistados aseguran que, hasta la fecha, les han llegado pocos mensajes negativos, y siempre se estudian antes de contestar o retirar. La clave del éxito en este caso está en la sinceridad, la planificación, la anticipación, el análisis y el sentido común, aseguran.

Con tanta sobreabundancia de información en los medios sociales, la atención se convierte en un recurso escaso en el mundo de los negocios, más que el talento y la tecnología. Por este motivo, el concepto de escucha al usuario en los medios sociales se considera un elemento importante. Prestar atención significa vigilancia o predisposición a percibir todo aquello que en el entorno pueda incrementar la información y el conocimiento. Igualmente, también significa que se debe estar atento para así poder resolver algún problema y simplemente asesorar al usuario. Aquí cada destino tiene su propia estrategia, basada en las informaciones compartidas por los usuarios. Varios de los destinos consultados tienen en cuenta también la influencia de los usuarios que comparten información, en previsión de las consecuencias. Es decir, la respuesta a un comentario está condicionada por la cantidad de seguidores de éste.

Los resultados de la encuesta a los gestores de los medios sociales son bastante coincidentes con las opiniones de los directores de comunicación. La mayoría de los destinos cuentan con una persona que se encarga de gestionar su presencia en los medios sociales. Su perfil podría describirse como el de mujer, de unos 37 años de edad, con conocimientos de informática y de turismo, mayoritariamente autodidacta, que busca, crea, monitoriza e introduce contenidos -con algún tipo de colaboración interna puntual - en 
las distintas redes sociales (sobre todo Facebook y Twitter) dirigidos especialmente a los turistas, siguiendo las directrices orientativas definidas de la dirección, como ya se ha comentado por los Directores de comunicación.

Un $69 \%$ de los gestores de los medios sociales encuestados coincide en señalar que en su destino dispone de una estrategia definida. En este sentido, los contenidos que más predominan, como se observa en el gráfico 2, son los relativos a la Información Turística/Agenda, aunque también destacan los contenidos sobre los Atractivos Naturales y el Patrimonio Tangible (monumentos, museos, etc.).

La mayoría de ellos se dirigen a turistas potenciales, siendo estos el principal público objetivo. Las empresas comercializadoras del propio destino son también públicos objetivos, coincidiendo con los resultados manifestados en las entrevistas en profundidad realizadas a los responsables de comunicación. Igual de importantes se consideran los turistas que los propios residentes del destino, que al contar con información actualizada de los diferentes recursos, atractivos y actividades de su destino, pueden convertirse en un altavoz de comunicación más a través de los medios sociales.

\section{Gráfico 1 \\ TIPO DE CONTENIDOS PUBLICADOS POR LOS DESTINOS TURÍSTICOS ANALIZADOS EN LOS MEDIOS SOCIALES}

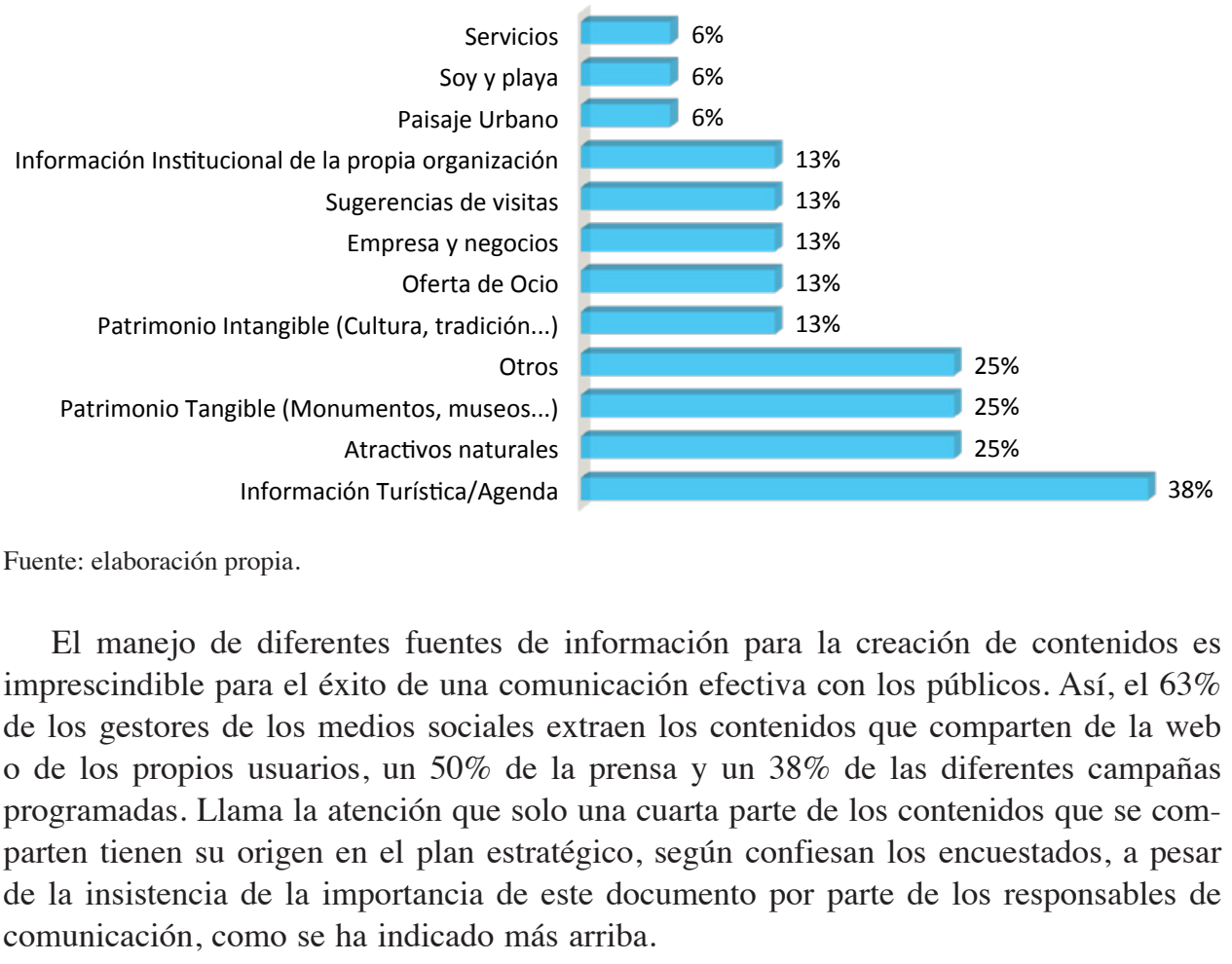

Cuadernos de Turismo, 41, (2018), 513-534 
Así pues, tanto los directores de comunicación como los gestores de los medios coinciden en la importancia de poseer una estrategia comunicativa para la comunicación a través de los medios sociales, pero en la práctica, los gestores reconocen que solo una cuarta parte de los contenidos surge de los planes estratégicos. Por otro lado, se reconoce el interés por la escucha a los comentarios de los usuarios, pero se trata de un interés mayoritario por conocer la influencia de estos usuarios y utilizar a los más influyentes en la difusión de sus contenidos que un interés por crear diálogo y relaciones con ellos. Finalmente, los contenidos más comunicados son algunos atractivos turísticos y temas de agenda, pero se deja de lado los valores emocionales de la marca del destino, a pesar de que los Directores de comunicación reconocen la importancia de su comunicación.

\subsection{Los contenidos y la proyección de la marca de los destinos en los medios sociales}

En este apartado se muestran los resultados obtenidos a partir del análisis de contenidos de las publicaciones realizadas por los destinos en los medios sociales. Como se ha señalado en la metodología, se analizaron 2759 publicaciones de 38 destinos turísticos españoles, catalogados según la clasificación establecida por la FEMP -alta montaña, ciudad media, ciudad patrimonial, destino interior, destino litoral, gran destino litoral y gran municipio- más cinco comunidades autónomas y el destino nacional en diferentes medios sociales -Facebook, Twitter, Flickr, Youtube y sus blogs-.

Para realizar este estudio, se tomaron diferentes categorías temáticas o atractivos turísticos y valores emocionales que ya se han detallado en la metodología. Así, en el análisis de contenido aparecen hasta 4855 conceptos mencionados, agrupados en las diferentes categorías.

Los medios sociales con más publicaciones de los destinos turísticos españoles, como ya indicaron los directores y los gestores de la comunicación digital, son Facebook, Twitter y Youtube. De los 2759 mensajes analizados, 953 se recogen en Facebook, lo que supone un 34\%; 781 en Twitter, es decir un 28\%; y 542 en Youtube, un 20\%. El resto fueron publicados en Flickr y en los blogs institucionales de los destinos.

Tabla 1

PORCENTAJE DE TEMAS MÁS COMPARTIDOS POR TIPO DE DESTINO

\begin{tabular}{|l|c|c|c|c|c|c|c|c|}
\hline & $\begin{array}{c}\text { Alta } \\
\text { montaña }\end{array}$ & $\begin{array}{c}\text { Ciudad } \\
\text { media }\end{array}$ & $\begin{array}{c}\text { Ciudad } \\
\text { patrimonial }\end{array}$ & $\begin{array}{c}\text { COMUNIDAD } \\
\text { /PAÍS }\end{array}$ & $\begin{array}{c}\text { Destino } \\
\text { interior }\end{array}$ & $\begin{array}{c}\text { Destino } \\
\text { litoral }\end{array}$ & $\begin{array}{c}\text { Gran destino } \\
\text { litoral }\end{array}$ & $\begin{array}{c}\text { Gran } \\
\text { municipio }\end{array}$ \\
\hline NATURALEZA & 48,55 & 4,14 & 7,05 & 23,97 & 15,88 & 9,12 & 17,30 & 3,40 \\
\hline TANGIBLES & 10,87 & 28,76 & 29,23 & 17,06 & 18,53 & 8,47 & 10,69 & 22,92 \\
\hline ESCAPADA & 7,97 & 13,51 & 15,90 & 13,51 & 10,29 & 5,54 & 4,09 & 21,22 \\
\hline INTANGIBLES & 7,25 & 19,17 & 15,26 & 11,58 & 20,88 & 15,64 & 15,41 & 11,04 \\
\hline OCIO & 13,77 & 24,40 & 18,97 & 8,97 & 25,59 & 13,03 & 8,18 & 27,67 \\
\hline SOLY PLAYA & 1,45 & 2,18 & 6,67 & 15,63 & 2,06 & 36,48 & 25,94 & 4,58 \\
\hline DEPORTE & 8,70 & 3,27 & 3,59 & 5,54 & 2,35 & 7,82 & 10,53 & 4,75 \\
\hline TECNOLOGÍA & 0,00 & 2,61 & 1,28 & 1,93 & 1,18 & 0,65 & 3,77 & 2,38 \\
\hline SERVICIOS & 1,45 & 1,96 & 2,05 & 1,81 & 3,24 & 3,26 & 4,09 & 2,04 \\
\hline
\end{tabular}

Fuente y elaboración propia. 
Respecto a los contenidos de las publicaciones, tal y como señalaban los responsables de comunicación y los gestores de los medios sociales y coincidiendo con ellos, los principales atractivos compartidos por los destinos turísticos son: la naturaleza, patrimonio tangible e intangible, escapadas, ocio, clima, deporte, negocio, tecnología y servicios.

Así, los destinos que más mensajes sobre naturaleza comparten en sus medios sociales son los de alta montaña $(48,55 \%)$, las comunidades autónomas $(23,97 \%)$, los grandes destinos litorales $(17,30 \%)$, y lógicamente, los destinos de interior $(15,88 \%)$. Sobre naturaleza los ítems más repetidos por todos los destinos son, entre otros, las escapadas a entornos naturales $(62,6 \%)$ y rurales $(14,9 \%)$, así como las menciones a la montaña $(14,4 \%)$ y al ecoturismo $(8,1 \%)$.

En el caso de los atractivos de patrimonio tangible los más mencionados por los diferentes destinos turísticos son: el patrimonio histórico $(48,2 \%)$, el patrimonio religioso $(12,5 \%)$, los museos $(11,7 \%)$ y las obras de arte $(11,4 \%)$. Por tipo de destino, los que más comparten estos contenidos son, en primer lugar, las ciudades patrimoniales $(29,23 \%)$ seguidos de cerca por las ciudades medias $(28,76 \%)$ y las grandes ciudades $(22,92 \%)$. También lo hacen, aunque en menor medida los destinos de interior $(18,53 \%)$ y las comunidades autónomas $(17,06 \%)$.

En el caso de los intangibles, la cultura popular $(41,9 \%)$, la gastronomía $(40,4 \%)$ y la cultura del vino $(17,2)$ también son temas que aparecen en la mayoría de los destinos estudiados, y en la mayoría de los casos en porcentajes similares, y que como en el caso anterior se trata de un elemento que complementa otras ofertas en cada una de las ciudades analizadas. Así, son los destinos de interior $(20,88 \%)$ y las ciudades medias $(19,17 \%)$ los que más mencionan este atractivo en sus medios sociales oficiales, seguidos de los destinos litorales, gran destino litoral y las ciudades patrimoniales, todas con más de un $15 \%$ de menciones.

Algo similar sucede con el ocio, que en líneas generales, es el segundo o el tercer tema más mencionado por todos los destinos menos en el caso de los grandes municipios $(27,67 \%)$ y los destinos de interior $(25,59 \%)$, en los que es el primero, ya que estos suelen apostar fuerte por sus fiestas y tradiciones. Le siguen las ciudades medias $(24,4 \%)$ y las patrimoniales $(18,97 \%)$. Los atractivos más repetidos relacionados con el ocio por las diferentes marcas turísticas en sus medios sociales son las escapadas urbanas y culturales $(71,6 \%)$, la vida nocturna $(18,6 \%)$ y shopping $(9,8 \%)$.

Cabe destacar que sólo los destinos litorales y grandes destinos litorales centran su estrategia de marca en la promoción de su buen clima, fundamentalmente del sol y playa. Un $36,48 \%$ y un $25,94 \%$ de sus mensajes, respectivamente, se refieren a este atractivo, que se concretan en destacar el clima en un $34,5 \%$ de las ocasiones y sus playas en un $48,7 \%$ de los casos. Otros temas destacados, pero con un número de menciones inferior al $6 \%$ son las actividades deportivas, el turismo tecnológico y la descripción de los servicios del destino.

Pasamos ahora a mostrar los valores emocionales de marca comunicados en las plataformas oficiales de los medios sociales analizados.

Como se observa, los valores emocionales más repetidos en el total de los 2759 mensajes analizados de los diferentes medios sociales y destinos turísticos son aquellos que se refieren a la honestidad -para referirse a lo tradicional, lo real y la tranquili- 
dad-, que se comunican en un $35,56 \%$ de las ocasiones. Le siguen lo imaginativo y lo enérgico, en ambos casos con un $21,13 \%$ de presencia en las publicaciones analizadas. Igualmente, los destinos apuestan por destacar entre sus valores emocionales el carácter saludable en un $16,53 \%$ de sus mensajes compartidos en los medios sociales y la alegría en un $14,61 \%$. Otros valores que comunican con frecuencia los destinos son el realismo $(12,47 \%)$, sus encantos $(11,13 \%)$, lo atrevido $(10,26 \%)$, su carácter cosmopolita $(6,45 \%)$, y lo exitoso $(5,11 \%)$.

Con todo, el estudio demostró que se comunican más y mejor los atractivos turísticos que los valores emocionales a través de los medios sociales. Por tanto, a pesar de que los directores de comunicación valoran la marca de destino como un activo importante en las entrevistas en profundidad, la realidad muestra que estos valores emocionales de marca no se recogen en general en los planes estratégicos ni en la estrategia de contenidos de los medios sociales, en los que se priorizan los atractivos turísticos y los temas de agenda.

\section{Gráfico 2 \\ VALORES EMOCIONALES MÁS COMUNICADOS EN LAS PUBLICACIONES DE LOS DESTINOS TURÍSTICOS EN SUS MEDIOS SOCIALES}

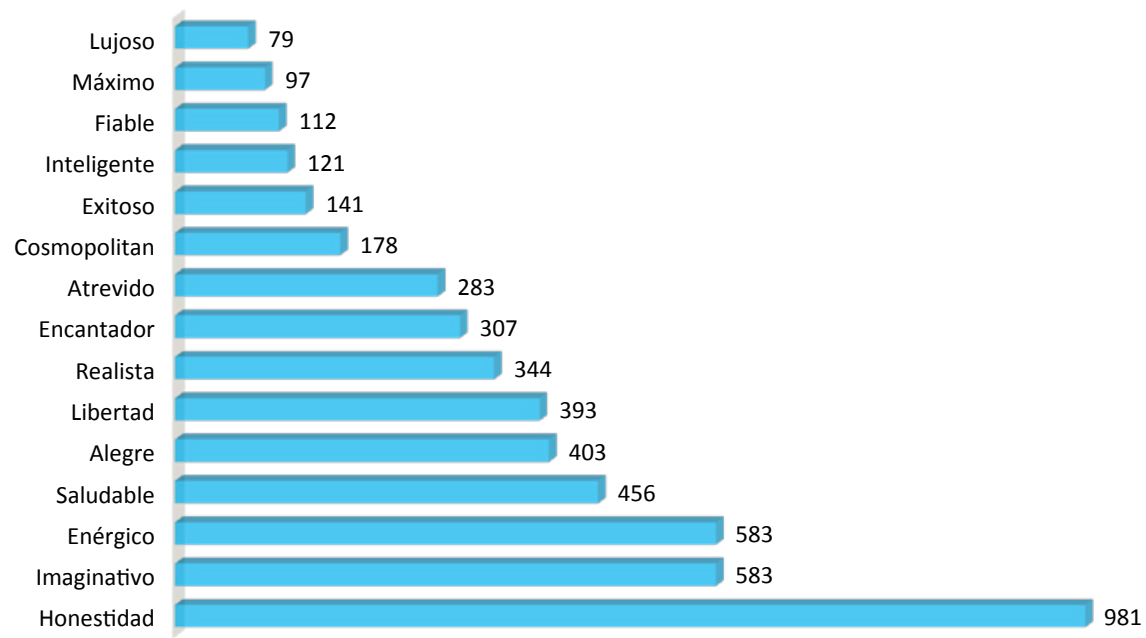

Fuente: elaboración propia.

\section{3. ¿Qué informaciones buscan los usuarios en los medios sociales?}

En este apartado se muestran los resultados de la tercera fase del estudio, que surgen de la encuesta que se realizó a usuarios españoles para conocer qué informaciones buscan cuando organizan un viaje y en qué medios sociales lo hacen.

La mayoría de los turistas tiene una o varias cuentas en las redes sociales, siendo Facebook la más utilizada, en un 91,6\%, seguida de Twitter con un 45\%, Instagram con un $32,9 \%$, LinkedIn con un $32 \%$, Youtube con un $30 \%$ y Google+ con un $29 \%$. 


\section{Gráfico 3 \\ MEDIOS SOCIALES MÁS UTILIZADOS POR LOS USUARIOS/TURISTAS}

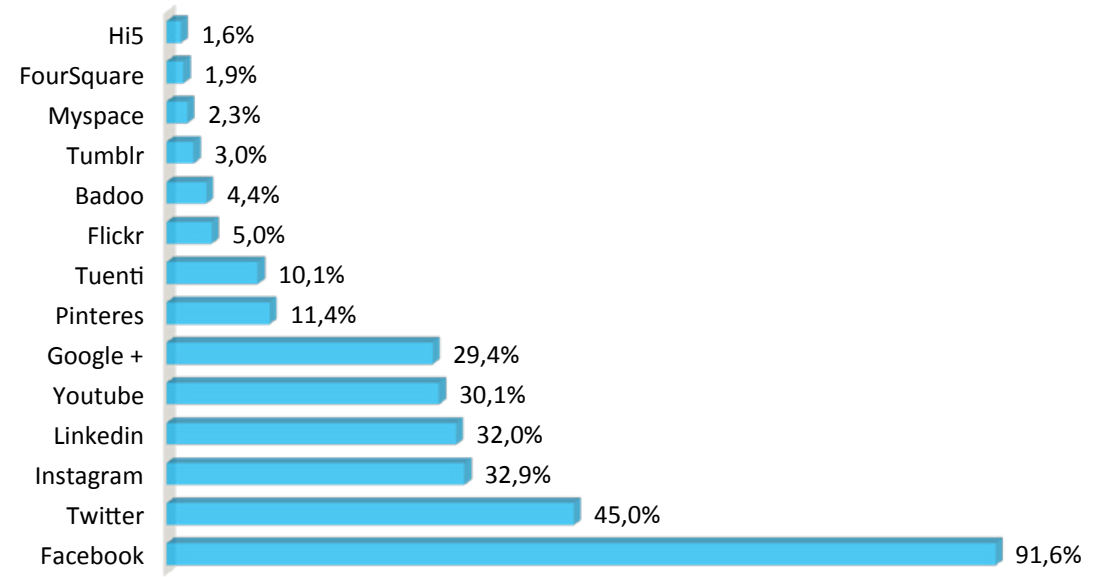

Fuente: elaboración propia.

De todas estas redes sociales, Facebook, Twitter e Instagram son las más utilizadas por los usuarios. En concreto, un $75 \%$ de los encuestados usa una o varias veces al día Facebook, mientras que casi un $15 \%$ lo hace varias veces a la semana. En el caso de Instagram, un $56 \%$ lo utiliza todos los días y un $21,9 \%$ varias veces a la semana. Twitter es usado todos los días por un $45,8 \%$ y un $21,9 \%$ varias veces a la semana. Casi el $80 \%$ de los encuestados, que reconoce acceder a las redes sociales, lo hace, fundamentalmente, a través de sus smartphones, aunque todavía muchos afirman hacerlo también mediante su portátil $(47,9 \%)$, su tablet (32\%) y el ordenador de mesa (41\%). Resulta curioso que en general los destinos turísticos españoles analizados utilicen más Flickr que Instagram como plataforma de compartición de fotografías cuando los usuarios utilizan mayoritariamente Instagram. Esto ya es un primer aspecto que deberían considerar.

La mayoría de los españoles encuestados $(59,1 \%)$ suele viajar en temporada de verano, aunque un $15,6 \%$ lo hicieron en primavera, un $13,4 \%$ en invierno y un $11,9 \%$ en otoño. Esto implica que la programación del trabajo de un destino turístico en las redes sociales debe realizarse a lo largo de todo el año, como se detalla más adelante.

Respecto a la elección del destino, más de la mitad de los encuestados $(55,4 \%)$ eligió un destino nacional, frente al 31,9\% que prefirió viajar por Europa. Un 3\% optó por visitar los Estados Unidos, un 5,4\% algún país de América Latina y un 2,3 Asia. La mayoría de los entrevistados que viajaron durante los últimos dos años contrataron sus vacaciones por cuenta propia en Internet y un 9,8\% lo hizo a través de una agencia online. Solo un 12,8\% contrató su estancia a través de una agencia de viajes.

De todos ellos, un $70 \%$ reconoce que antes de elegir un destino buscó información en Internet sobre alojamientos $(16 \%)$, excursiones $(11,3 \%)$, actividades de ocio y compras $(5,4 \%)$, restauración $(4,6 \%)$, información sobre cultura, museos y exposiciones $(7,6 \%)$, principales atractivos turísticos $(28,8 \%)$ y lugares más visitados $(10,3)$. Esta búsqueda de información la 
realizaron en la web oficial del destino $(14,2 \%)$, en una web de viajes $(13,1)$, en buscadores $(26,1 \%)$, en blogs de viaje $(10,1 \%)$, en portales de recomendación como Tripadvisor $(11,2 \%)$ o en las redes sociales $(2,8 \%)$ para buscar información antes de contratar el viaje.

De igual manera, un $71 \%$ de los turistas encuestados aseguran que también buscan información durante su estancia en el destino elegido. En este caso, suelen utilizar fundamentalmente buscadores, la web oficial del lugar y medios sociales para localizar los principales atractivos turísticos $(18,5 \%)$, zonas de restauración $(18,3 \%)$ y excursiones $(17,2 \%)$.

De los que optaron por utilizar los medios sociales para obtener información que les ayudase a organizar un viaje, la mayoría buscó en Facebook. Así lo confiesa un 73,9\% de los encuestados, frente al 23,2 que lo hizo en Google+ \%, el 15,9\% que también usó Youtube o el $13 \%$ que lo hizo en Twitter. Durante su estancia en el lugar sigue siendo Facebook la red social preferida para informarse, seguida de Google+, y Twitter, que desbanca a Youtube el tercer puesto en este momento.

Por su parte, el $65,1 \%$ de los encuestados comparten información sobre su viaje a la vuelta de las vacaciones. Un $66,8 \%$ suele recomendar los lugares visitados y solo un $14,2 \%$ publica alguna queja o denuncia en los medios sociales, bien en sus perfiles personales o en las cuentas oficiales de los lugares visitados. Para ello, eligen de nuevo Facebook $(81,1 \%)$ como medio principal, seguido, en este caso de Instagram $(23,6 \%)$ y Twitter $(13,8 \%)$.

En cuanto a la credibilidad que les ofrecen estos canales de comunicación a los viajeros, destaca Tripadvisor como canal en el que confía el 26,1\% de los encuestados, las páginas de Facebook oficiales, que resultan fiables para un 18,9\% y los blogs de viaje (13\%) y de destinos (10\%). Llama la atención que un $9 \%$ de los encuestados también confía en la información sobre un destino que comparten en Facebook amigos, familiares y cuentas no oficiales, y que este porcentaje aumenta entre públicos más jóvenes.

Así pues, comparando estos resultados con los análisis previos, se observa que los destinos turísticos utilizan los mismos medios sociales que los usuarios cuando estos van a buscar información u organizar un viaje: Facebook, Twitter y Youtube, por este orden. Pero con alguna excepción. Mientras los destinos españoles analizados poseen mayoritariamente cuentas de Flickr para compartir sus fotografías, los usuarios utilizan más Instagram con este fin. En cuanto a los contenidos, los destinos comunican mayoritariamente: actividades de ocio y agenda, patrimonio tangible, y otros atractivos turísticos, que son principalmente los contenidos buscados por los usuarios para la organización de viajes. En cambio, ofrecen poca información sobre gastronomía y restauración o sobre compras, que sí son temas buscados por los usuarios. Así pues, creemos que sería muy recomendable que los gestores de la comunicación de los destinos turísticos estuvieran más atentos a las necesidades de información de los usuarios en los medios sociales para que su comunicación de marca a través de los medios sociales sea más estratégica.

\section{DISCUSIÓN}

Por lo que respecta a las estrategias comunicativas, la mayoría de los directores de comunicación de los destinos turísticos destacan la importancia de implicar a los diferentes stakeholders -turistas, profesionales del sector y residentes- en la construcción de la marca. Todos coinciden en afirmar, que para ello se necesita una buena coordinación, que 
normalmente recae en la dirección de comunicación de las instituciones turísticas. Con todo, a pesar de la importancia que le confieren a la marca, los resultados muestran que esta es poco prioritaria en la gestión de los contenidos publicados en los medios sociales, especialmente los valores emocionales de marca, que se comunican poco y de forma no distintiva. Ello resulta sorprendente si tenemos en cuenta los estudios que demuestran que las marcas aportan un valor relacional con los públicos y ayudan a que los consumidores se identifican con su personalidad (Hankinson, 2004). Si además se conoce el potencial que poseen los medios sociales para comunicar las marcas (Kasemsap, 2016) y conseguir que los usuarios establezcan relaciones emocionales con ellas (Zaglia, 2013), la poca comunicación de las marcas y sus valores emocionales por los destinos resulta todavía más asombroso.

Para mejorar la planificación, todos los destinos cuentan con diferentes protocolos de actuación: planes estratégicos de turismo y planes de comunicación y marketing. Así lo confirman los gestores de los medios sociales también en las encuestas, que coinciden en señalar en un $69 \%$ de los casos que en su destino se dispone de una estrategia definida. El $63 \%$ de los gestores de los medios sociales extraen los contenidos que publican de la web, un $50 \%$ de la prensa y un $38 \%$ de las diferentes campañas programadas. Llama la atención que solo una cuarta parte de los contenidos que se comparten tienen su origen en el plan estratégico. Así pues, parece que las estrategias comunicativas y los planes estratégicos son más documentos de imagen que herramientas útiles de acción que guían la gestión comunicativa en los medios sociales. Otra vez sorprende que a pesar de que muchos estudios afirman la necesidad de conocer el funcionamiento de las marcas en los medios sociales (Habibi et al., 2014) y de establecer estrategias como la creación de comunidades de marca (Park y Kim, 2014), la planificación estratégica no se aplique en la gestión de los medios sociales.

Facebook, Twitter, Youtube, Flickr, Foursquare y Google + son los principales medios sociales en los que tienen presencia los destinos estudiados, si bien los más utilizados son Facebook, Twitter y Youtube, por este orden, al igual que muestran los resultados de otros estudios (Roque y Raposo, 2015). Y las razones por las que se eligieron estos canales son fundamentalmente para mejorar la difusión de la marca, para tener seguidores o fans y porque se requiere una menor inversión que en otros medios. Como se ha observado, los destinos y los usuarios coinciden en utilizar los tres principales medios sociales, pero difieren en los menos utilizados. Así, por ejemplo, mientras la mayoría de destinos turísticos posee cuentas de Flickr, los usuarios prefieren mayoritariamente Instagram. Como consecuencia, es necesario que los destinos analicen los usos de los medios sociales por parte de los usuarios para orientarse mejor en cuáles de ellos estar presentes y conseguir así una comunicación más efectiva con los usuarios/turistas.

La mayoría de destinos analizados se proponen en su estrategia digital objetivos cuantitativos (como incrementar el número de seguidores y ganar visibilidad) y en algunos casos, la interacción y el valor cualitativo de las opiniones sobre la marca, así como la segmentación de los mensajes y los análisis. Pero se ha observado en los resultados que, en realidad, la marca y sus valores emocionales se comunican menos que la agenda o ciertos atractivos turísticos. Esto es coincidente con los resultados de estudios anteriores (Bigné, García y Blas, 2009; Michaelidou et al., 2013; Míguez y Huertas, 2015). Así pues, los destinos turísticos utilizan los medios sociales como canales de información para comu- 
nicar los temas que les parecen relevantes sin tener demasiado en cuenta la comunicación de la marca y tampoco las opiniones y las necesidades de información de los usuarios.

En general existe una coherencia entre lo que publican los destinos y lo que buscan los usuarios. Son los destinos de alta montaña, los destinos de interior y los grandes destinos litorales los que más apuestan por los atractivos naturales. Las ciudades medias optan por centrar su estrategia en la promoción de su patrimonio tangible, el ocio y el patrimonio intangible, fundamentalmente aquellos aspectos tradicionales e históricos. Los destinos de ciudades patrimoniales, como es lógico destacan en los medios sociales por sus contenidos relacionados con sus monumentos, museos, el ocio y sus actividades nocturnas, relacionadas con la actividad cultural. Los destinos litorales, tanto los pequeños como los grandes, apuestan por destacar entre sus atractivos su buen clima, el sol y la playa, la naturaleza y el ocio. Con todo, también se observa que existe algún atractivo buscado por los usuarios, como: alojamiento, gastronomía, restauración o compras, que son poco comunicados por los medios sociales de los destinos.

Siete de cada diez turistas reconoce que antes de elegir un destino busca información en Internet, sobre todo en buscadores, la página web oficial de un lugar concreto y en páginas y blogs de viaje y los medios sociales. Fundamentalmente indagan sobre alojamientos (16\%), excursiones $(11,3 \%)$, actividades de ocio y compras $(5,4 \%)$, restauración $(4,6 \%)$, información sobre cultura, museos y exposiciones $(7,6 \%)$, principales atractivos turísticos $(28,8 \%)$ y lugares más visitados (10,3). Casi el mismo porcentaje de viajeros hacen lo propio cuando ya están en el destino, si bien en esta ocasión requieren información sobre restauración, atractivos turísticos y excursiones. Por todo ello, se recomienda que los destinos escuchen más las necesidades de los turistas en los medios sociales y adapten más su comunicación a estas necesidades si quieren realizar una comunicación efectiva con sus públicos.

\section{CONCLUSIONES Y PROPUESTAS}

A partir del primer objetivo de nuestro estudio, que era conocer si los destinos utilizan los medios para crear diálogo con los usuarios y generar una buena imagen, podemos concluir que las OMDs deberían incentivar más la interacción con los usuarios a través de los medios sociales, por ejemplo, realizando preguntas para conocer mejor la opinión de los viajeros y sus necesidades de información y poder así segmentar y personalizar más su estrategia comunicativa.

La personalización de la información y de los productos turísticos son el futuro; los destinos deben programar los productos a medida, en función del tipo de público, y sus diferentes necesidades: viajar con niños, jóvenes, sol y playa, cultura, escapadas, etc.; y los medios sociales son una canal importante para segmentar la oferta. Por ello es importante conocer exactamente a través de qué medios sociales llegar a cada tipo de público y conocer también con exactitud qué informaciones los usuarios buscan en cada medio. Así pues, los medios sociales deben seleccionarse cuidadosamente, así como el uso de cada uno y los contenidos a publicar.

Respecto al objetivo de conocer si las informaciones que buscan los usuarios en los medios sociales coinciden con los contenidos de la comunicación de los destinos, podemos concluir que en general así es, aunque también se observa que existe algún tema buscado por 
los usuarios, como: alojamiento, gastronomía, restauración o compras, que son poco comunicados por los medios sociales de los destinos. Así pues, las estrategias comunicativas de las OMDs deberían estar más ajustadas a las necesidades comunicativas de los usuarios o turistas.

Es importante tener en cuenta las fases del viaje de los usuarios y sus necesidades de información en la comunicación a través de los medios sociaes; ya que no es lo mismo la información que buscan antes del viaje, la que necesitan durante su estancia en un destino o sus comentarios y valoraciones a la vuelta. En muchas ocasiones las OMDs no tienen en cuenta estas fases del viaje en su estrategia comunicativa y ello es clave para que los contenidos publicados en cada medio sean realmente estratégicos en cuanto al momento de publicación y búsqueda.

Respecto al último objetivo del estudio de analizar la correcta comunicación de las marcas de destino a través de los medios sociales con sus dos componentes principales, puede afirmarse que en muchas ocasiones la marca no está presente en los contenidos publicados por las OMDs a pesar de contar casi todos los destinos con estrategias de comunicación. Con todo, las estrategias de marca no abundan y finalmente se acaban comunicando más y mejor los atractivos turísticos que los valores emocionales. Las OMDs deben potenciar las estrategias de comunicación de marcas, establecer directrices claras de comunicación al respecto y conseguir que los valores emocionales se comuniquen de igual modo a través de los medios sociales. Ello es clave si se quieren poseer marcas de destino bien posicionadas y reconocidas.

Para que todo ello sea posible, hay que destacar la necesidad de contar con profesionales especializados en comunicación digital. El perfil ideal es aquel que sume habilidades propias para los medios sociales (inmediatez, interacción, alerta de novedades, gestión de incertidumbres) y comunicativas (planificación, comunicación estratégica, conocimiento de los públicos, y voluntad expresa de comunicación de la marca de destino).

Las discusiones y conclusiones de este estudio han supuesto una contribución importante a la comunicación de los destinos turísticos a través de sus medios sociales. Han mostrado los puntos flojos en la práctica comunicativa de la profesión y han destacado los aspectos claves que las OMDs deben tener en cuenta para conseguir una comunicación efectiva con sus usuarios.

Con todo, este estudio también posee limitaciones. La comparación de resultados provenientes de cuatro metodologías de análisis distintas centradas en diversos públicos, que incluyen desde entrevistas en profundidad a los directores de comunicación, encuestas a los gestores, encuestas a usuarios y análisis de contenido de los medios sociales, supone una gran dificultad y evidentes limitaciones. Por otro lado, el análisis de contenido de los medios sociales se realizó manualmente, lo cual comporta problemas de representatividad de todos los contenidos publicados.

\section{REFERENCIAS}

AAKER, J.L. (1997): «Dimensions of Brand Personality», Journal of Marketing Research, $n^{\circ} 34$, pp. 347-356. 
ALGESHEIMER, R., DHOLAKIA, U.M., y HERRMANN, A. (2005): «The social influence of brand community: Evidence from European car clubs», Journal of Marketing, $\mathrm{n}^{\circ} 69(3)$, pp. 19-34.

BALOGLU, S. y McCLEARY, K.W. (1999): «A Model of Destination Image Formation», Annals of Tourism Research, $\mathrm{n}^{\circ}$ 26, pp. 868-897.

BEERLI, A. y MARTIN, J. D. (2004): «Factors influencing destination image», Annals of tourism research, $\mathrm{n}^{\mathrm{o}} 31$ (3), pp. 657-681.

BIGNÉ, E.J., M.I. SÁNCHEZ GARCÍA y SANZ BLAS, S. (2009): «The FunctionalPsychological Continuum in the Cognitive Image of a Destination. A Confirmatory Analysis», Tourism Management, $\mathrm{n}^{\mathrm{o}} 30$ (5), pp. 715-723.

BLAIN, C., LEVY, S.E. y RITCHIE J.R.B. (2005): «Destination Branding: Insights and Practices from Destination Management Organizations», Journal of Travel Research, no 43(4), pp. 328-38.

BRODIE, R.J., ILIC, A., JURIC, B., y HOLLEBEEK, L. (2013): «Consumer engagement in a virtual brand community: An exploratory analysis», Journal of Business Research, $\mathrm{n}^{\circ} 66$ (1), pp. 105-114.

CHOI, S., LEHTO, X.Y. y MORRISON, A.M. (2007): «Destination image representation on the web: Content analysis of Macau travel related websites», Tourism Management, $\mathrm{n}^{\circ} 28$, pp. 118-129.

DE MOYA, M. y JAIN, R. (2013): «When tourists are your "friends": Exploring the brand personality of Mexico and Brazil on Facebook», Public Relations Review, $\mathrm{n}^{\circ}$ 39, pp. 23-29.

DICKINGER, A. y LALICIC, L. (2016): «An analysis of destination brand personality and emotions: a comparison study», Information Technology \& Tourism, $\mathrm{n}^{\circ} 15$ (4), pp. 317-340.

ECHTNER, C.M. y RITCHIE, J.R.B. (2003): «The Meaning and Measurement of Destination Image», Journal of Tourism Studies, $\mathrm{n}^{\circ} 14$ (1), pp. 37-48.

EKINCI, Y. y HOSANY, S. (2006): «Destination Personality: An Application of Brand Personality to Tourism Destinations», Journal of Travel Research, $\mathrm{n}^{\circ}$ 45, pp. 127-139.

FEMP y TOURSPAIN (Eds.) (2008): Modelos de gestión turística local: Principios y prácticas. Federación Española de Municipios y Provincias, Secretaría General de Turismo, Madrid, España.

FOURNIER, S. (1998): «Consumers and Their Brands: Developing Relationship Theory in Consumer Research», Journal of Consumer Research, $\mathrm{n}^{\circ}$ 24, pp. 343-73.

GALLARZA, M.G., SAURA, I.G. y GARCÍA, H.C. (2002): «Destination image: Towards a conceptual framework», Annals of Tourism Research, no 29 (1), pp. 56-78.

GOVERS R. y GO, F. M. (2003): «Deconstructing destination image in the information age», Information Technology and Tourism, $\mathrm{n}^{\circ} 6$ (1), pp. 13-29.

GOVERS, R. y GO, F.M. (2009): Place branding: Glocal, virtual and physical identities, constructed, imagined and experienced. Hampshire, Palgrave Macmillan.

GOVERS, R., GO, F. M., y KUMAR, K. (2007): «Promoting tourism destination image», Journal of Travel Research, $\mathrm{n}^{\circ} 46$ (1), pp. 15-23.

GRUNIG, J. E., y HUNT, T. (1984): Managing public relations ( $\left.\mathrm{n}^{\mathrm{o}} 343\right)$. New York: Holt, Rinehart and Winston. 
HABIBI, M.R., LAROCHE, M., y RICHARD, M.O. (2014): «The roles of brand community and community engagement in building brand trust on social media», Computers in Human Behavior, $\mathrm{n}^{\circ} 37$, pp. 152-161.

HANKINSON, G. (2004): «Relational network brands: Towards a conceptual model of place brands», Journal of Vacation Marketing, n ${ }^{\circ} 10$ (2), pp. 109-121.

HAYS, S., PAGE, S.J. y BUHALIS, D. (2013): «Social media as a destination marketing tool: its use by national tourism organisations», Current issues in tourism, $\mathrm{n}^{\circ} 16$ (3), pp. 211-239.

HENNING-THURAU, T., GWINNER, K.P, WALSH, G. y GREMLER, D. (2004): «Electronic word-of-mouth via consumer-opinion platforms: What motivates consumers to articulate themselves on the Internet?», Journal of Interactive Marketing, $\mathrm{n}^{\mathrm{o}} 18(1)$, pp. 38-52.

HOSANY, S., EKINCI, Y. y UYSAL, M. (2006): «Destination Image and Destination Personality: An Application of Branding Theories to Tourism Places», Journal of Business Research, $\mathrm{n}^{\circ}$ 59, pp. 638-642.

HUERTAS, A. (2014): La comunicación de los territorios, los destinos y sus marcas. Guía práctica de aplicación desde las relaciones públicas. Barcelona, DIRCOMUOC.

HUERTAS, A. y MARINÉ-ROIG, E. (2015): «Destination Brand Communication Through the Social Media: What Contents Trigger Most Reactions of Users?» en Tussyadiah y Inversini (Eds.), Information and Communication Technologies in Tourism 2015. Proceedings of the International Conference in Lugano, Switzerland. Vienna: Springer.

HUERTAS, A., SETÓ, D. y MíGUEZ, M.I. (2015): «Comunicación de destinos turísticos a través de los medios sociales», El Profesional de la Información, nº 24 (1), pp. 15-21.

HVASS, K.A. y MUNAR, A.M. (2012): «The takeoff of social media in tourism», Journal of Vacation Marketing, n 18 (2), pp. 93-103.

INVERSINI, A. y BUHALIS, D. (2009): «Information convergence in the long tail: The case of tourism destination information», en W. Hopken, U. Gretzel, y R. Law (Eds.), Information and Communication Technologies in Tourism 2009. Vienna, Springer, pp. 381-392.

KASEMSAP, K. (2016): «Role of Social Media in Brand Promotion: An International Marketing Perspective». En Managing Public Relations and Brand Image through Social Media (pp. 62-88). IGI Global.

LAROCHE, M., HABIBI, M.R. y RICHARD, M.O. (2013): «To be or not to be in social media: How brand loyalty is affected by social media?», International Journal of Information Management, $\mathrm{n}^{\mathrm{o}} 33$, pp. 76-82.

LEUNG, D., LAW, R, VAN HOOF, H. y BUHALIS, D. (2013): «Social media in tourism and hospitality: A literature review», Journal of Travel \& Tourism Marketing, $\mathrm{m}^{\mathrm{o}} 30(1-2)$, pp. 3-22.

MARCHIORI, E. y CANTONI, L. (2012): «The online reputation construct: Does it matter for the tourism domain? A literature review on destinations' online reputation», Journal of Information Technology \& Tourism, nº 13 (3), pp. 139-159. 
MARINÉ-ROIG, E. (2013): «From the projected to the transmitted image: the 2.0 construction of tourist destination image and identity in Catalonia», PhD dissertation. Retrieved from http://hdl.handle.net/10803/135006

MICHAELIDOU, N., SIAMAGKA, N.T., MORAES, C. y MICEVSKI, M. (2013): «Do Marketers Use Visual Representations of Destinations That Tourists Value? Comparing Visitors' Image of a Destination with Marketer-Controlled Images Online», Journal of Travel Research, $\mathrm{n}^{\circ} 52$ (6), pp. 789-804.

MÍGUEZ, M.I. y HUERTAS, A. (2015): «The power of photograph in the communication and public relations of tourist destinations and their brands through Facebook and Flikr», Catalan Journal of Communication and Cultural Studies, $\mathrm{n}^{\circ} 7$ (2), pp. 197-215.

MILL, R.C. y MORRISON, A.M. (2002): The tourism system. Dubuque, Iowa, Kendall.

MORGAN, N.J., PRITCHARD, A. y PIGGOTT, R. (2003): «Destination branding and the role of the stakeholders: The case of New Zealand», Journal of Vacation Marketing, $\mathrm{n}^{\circ} 9$ (3), pp. 285-299.

MUNAR, A.M. (2011): «Tourist-created content: rethinking destination branding», International Journal of Culture, Tourism and Hospitality Research, $\mathrm{n}^{\circ} 5$ (3), pp. 291-305.

PIKE, S. (2002): «Destination Image Analysis-A Review of 142 Papers from 1973 to 2000», Tourism Management, $\mathrm{n}^{\circ}$ 23, pp. 541-49.

PIKE, S. y RYAN, C. (2004): «Destination positioning analysis through a comparison of cognitive, affective and conative perceptions», Journal of Travel Research, $\mathrm{n}^{\circ}$ 42, pp. 333-342.

ROQUE, V. y RAPOSO, R. (2015): «The usage of social media by the destination management organizations. The case study of the Portuguese DMO, Dos Algarves. A Multidisciplinary E-journal, $\mathrm{n}^{\circ} 26$ (2), pp. 87-114.

SENECAL, S. y NANTEL, A. (2004): «The influence of online product recommendations on consumers' online choices», Journal of Retailing, no 80 (2), pp. 159-169.

SIRGY, M.J. (1982): «Self-concept in Consumer-Behaviour: A Critical Review», Journal of Consumer Research, $\mathrm{n}^{\circ}$ 9, pp. 287-300.

SCHMALLEGGER, D. y CARSON, D. (2010): «Destination image projection on consumer generated content websites (CGC): a case study of the Flinders Ranges», Journal of Information Technology \& Tourism, $\mathrm{n}^{\mathrm{o}} 11$ (2), pp. 111-127.

STEPCHENKOVA, S. y ZHAN, F. (2013): «Visual destination images of Peru: Comparative content analysis of DMO and user-generated photography», Tourism Management, $\mathrm{n}^{\circ} 36$, pp. 590-601.

THEVENOT, G. (2007): «Blogging as a social media», Tourism and hospitality research, $\mathrm{n}^{\mathrm{o}} 7$ (3-4), pp. 287-289.

TUSSYADIAH, I.P. y FESENMAIER, D.R. (2009): «Mediating tourist experiences: Access to places via shared videos», Annals of Tourism Research, $\mathrm{n}^{\circ} 36$ (1), pp. 24-40.

WENGER, A. (2008): «Analysis of travel bloggers' characteristics and their communication about Austria as a tourism destination», Journal of Vacation Marketing, $\mathrm{n}^{\mathrm{o}} 14$ (2), pp. 169-176. 
WIGLEY, S. y LEWIS, B. K. (2012): «Rules of engagement: Practice what you tweet», Public Relations Review, $\mathrm{n}^{\mathrm{o}} 38$ (1), pp. 165-167.

XIANG, Z. y GRETZEL, U. (2010): «Role of social media in online travel information search», Tourism Management, $\mathrm{n}^{\circ} 31$ (2), pp. 179-188.

YOO, K. H. y GRETZEL, U. (2011): «Antecedents and impacts of trust in travel-related consumer-generated media», Journal of Information Technologies \& Tourism, $\mathrm{n}^{\circ} 12$ (2), pp. 139-152.

ZAGLIA, M.E. (2013): «Brand communities embedded in social networks», Journal of business research, $\mathrm{n}^{\circ} 66$ (2), pp. 216-223. 